\title{
Evaluation and establishment of a canine model of delayed splenic rupture using contrast-enhanced ultrasound
}

\author{
JIANGKE TIAN ${ }^{1}$, XIA XIE $^{1}$, FAQIN LV ${ }^{1}$, TENGFEI YU ${ }^{1}$, RONG WU ${ }^{1}$, \\ XINGHUA ZHANG ${ }^{2}$, DONG WANG ${ }^{1}$ and JIE TANG $^{1}$ \\ Departments of ${ }^{1}$ Ultrasound and ${ }^{2}$ Radiology, Chinese People's Liberation \\ Army General Hospital, Beijing 100853, P.R. China
}

Received March 29, 2012; Accepted June 8, 2012

DOI: $10.3892 / \mathrm{mmr} .2012 .948$

\begin{abstract}
The aim of the present study was to establish a canine model of delayed splenic rupture (DSR). A total of 15 mongrel dogs were anesthetized and laparotomized. The hematomas were observed following an injection of heparin. The hematomas were ruptured. The severity of the spleen rupture was evaluated and the intra-abdominal free liquid was measured. The splenic hematomas in the dogs continued to form and the hematoma area gradually increased. The hematomas were ruptured after impacting the abdominal wall. The spleens were damaged, and conventional ultrasonography showed intra-abdominal free liquid. These conditions were demonstrated via computed tomography scanning. A DSR canine model was established successfully.
\end{abstract}

\section{Introduction}

Delayed splenic rupture (DSR) is a rare type of splenic injury, which was first reported by Baudet in 1902. Baudet described the syndrome of delayed post-injury splenic rupture, in which the patient has a latent period with few or no symptoms immediately after the trauma, but develops evidence of intraabdominal hemorrhage one or more days later $(1,2)$. DSR is a rare but well-recognized clinical entity, which has been defined as the late occurrence of symptoms and signs in patients experiencing no initial hemodynamic instability or clinical symptoms $48 \mathrm{~h}$ or more after injury (3). DSR contributes to a significant mortality rate $(5-15 \%)(1,4)$ compared with that of acute splenic rupture $(1 \%)(5,6)$. Thus, its rapid diagnosis is urgently required.

The management and diagnosis of DSR is a result of collective investigations, in which a physical examination

Correspondence to: Dr Jie Tang, Department of Ultrasound, Chinese People's Liberation Army General Hospital, 28 Fuxing Road, Beijing 100853, P.R. China

E-mail: tangjiebeijing@126.com

Key words: delayed splenic rupture, animal model, ultrasound, contrast enhanced ultrasound used to be the first workup. Since the mid-1980s, however, computed tomography (CT) scanning has become a mainstay in the evaluation of intra-abdominal injury in hemodynamically stable patients. The term 'delayed splenic rupture' used before the CT scan era simply referred to a delayed diagnosis of splenic injury that evolved into a rupture. Isolated reports of trauma patients with initially normal CT scans and delayed splenic rupture have been published since the routine use of CT scan.

Some patients with mild symptoms and negative CT results underwent a non-operative management and were discharged from the hospital. However, they returned after a few days with hypovolemic shock, and the recurring CT scan showed a subcapsular splenic hematoma. The most important factor for the DSR was that little attention had been paid to the delayed development of splenic hematoma, particularly in the period between the hospital discharge and the subsequent rupture.

Nevertheless, a CT scan is usually not the first technique used in the diagnosis of DSR, although it has certain advantages. Ultrasonography (US) plays an important role in assessing the traumatized spleen, in view of its being both easily accessibile and cost-effective, hence delivering rapid results in screening. It has been widely used when detecting abdominal free fluid in patients with abdominal trauma due to its high sensitivity and availability, even though it cannot reliably determine the exact site of the active hemorrhage (7-9). On the other hand, CT has been proven to be efficient for trauma evaluation. However, the patient requires exposure to X-irradiation and needs to be removed from the emergency department $(10,11)$. In addition, contrast-enhanced ultrasound (CEUS) has been improved greatly and applied widely. It shows the extent and the size of intra-abdominal injury and active bleeding, which has been demonstrated in various animal experiments and clinical studies (12).

The experimental outline of the current study was inspired by the following points: i) little attention has been paid to the delayed development of a splenic hematoma; ii) DSR is extremely dangerous and requires immediate medical attention; iii) the study provides a canine model with DSR for additional investigation in the diagnosis and treatment of DSR.

The current study on canines was delineated to monitor the formation, development and breakdown of the splenic hematoma with the guidance of CEUS. 


\section{Materials and methods}

Animal model for hematomas of the spleen. All experiments abided by the guidelines issued by the National Institute of Health for the Care of Laboratory Animals and were performed according to a protocol approved by the Animal Care and Use Committee of our institution.

A total of 15 mongrel dogs, aged 2-3 years and weighing $18-22 \mathrm{~kg}$, with a health certificate [license number: SYXK (Beijing) 2007-0004] were used in the current study. General anesthesia was induced via intravenous injection of $30 \mathrm{mg} / \mathrm{kg}$ pentobarbital sodium (3\%) and was maintained by intramuscular injection of $5 \mathrm{mg} / \mathrm{kg}$ pentobarbital sodium. Trauma was not induced until the dogs were successfully anesthetized. The spleen was then exteriorized from the peritoneal cavity by a median laparotomy leaving intact the vascular pedicle. At this point, the organ was directly inspected to rule out other pathologies or already existing subcapsular hematomas. In the center of the organ, a hematoma was created by pinching both sides of the spleen (Fig. 1). The needle (16-gauge) was inserted into the hematoma through the normal splenic tissue. Consequently, the tissue inside the hematoma was damaged by swinging the needle and causing a hemorrhage. Heparin (5000 U) was injected into the hematoma through the normal spleen tissue (Fig. 2), and was used to maintain the continuous bleeding status in the organ. Moreover, $\alpha$-cyanoacrylate $(1 \mathrm{ml}$; Guangzhou Baiyun Medical Adhesive Co., Ltd., Guangzhou, China) was injected into the pathway to close the puncture in the normal splenic tissue. Surgicel (Johnson \& Johnson, USA) was pressed onto the surface of the spleen to avoid possible bleeding at the puncture point (Fig. 3), and the abdominal incision was closed in layers. Conventional US and CEUS were performed subsequent to creating a hematoma. The location, shape, size and sonographic appearance of the hematoma were registered, along with the area of the hematoma. An intravenous drip of heparin $(200 \mathrm{U} / \mathrm{kg} / 8 \mathrm{~h}$, diluted by $5 \%$ sodium chloride) was administered in order to maintain the anticoagulation.

Animal model for delayed splenic rupture. After $72 \mathrm{~h}$, the hematoma was ruptured by impacting the abdominal wall of the dogs using the impacting device (Fig. 4). The impacting device consisted of a supporter, an impacting handle, a piston handle, a power bullet and a power-actuated fastening device (13). The impacting force was recorded by a mechanical force transducer data recorder. After the bullet was loaded, the power-actuated fastening device was fixed into the supporter. The impacting handle was then inserted into the gun barrel, aimed at the splenic region and the trigger was pulled. The force of the bullet pushed the piston handle and the impacting handle onto the designated target region, which was located using the conventional sonography before impact. The impacting force was calculated based on the weight of the dog at $0.28 \mathrm{kN} / \mathrm{kg}$. In the current experiment, the force was $4.8-5.6 \mathrm{kN}$ (the mean, $5.3 \pm 0.3 \mathrm{kN})$.

Conventional US, CEUS and CT were performed to observe the conditions prior and subsequent to rupturing the hematoma, as well as the hemorrhage. Splenectomy was performed, the spleen specimens were harvested and evaluated by gross examination, after observation.

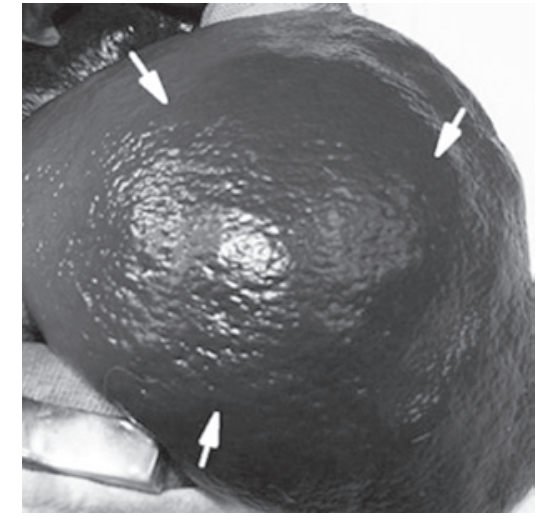

Figure 1. Hematoma of the spleen. A hematoma was created by pinching both sides of the spleen (white arrows).

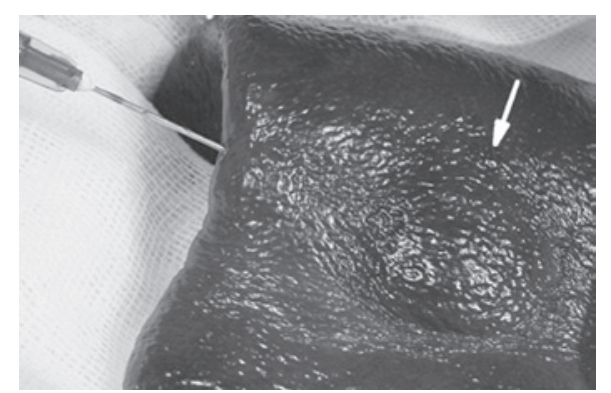

Figure 2. Heparin was injected into the hematoma through the normal spleen tissue (white arrow).

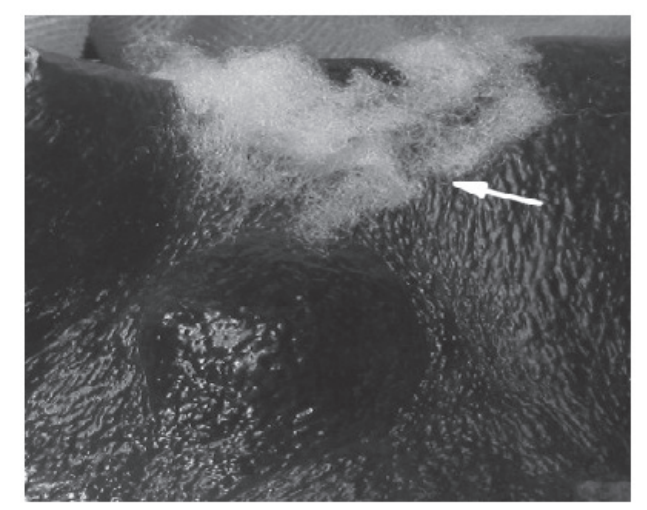

Figure 3. Surgicel was pressed onto the surface of the spleen to avoid possible bleeding at the puncture point (white arrow).

US contrast agent. The US contrast agent used in the current study was SonoVue (Bracco, Milan, Italy), a suspension of stabilized sulfur hexafluoride (SF6) microbubbles in saline (14-17). The bubble concentration is in the range of $1 \times 10^{8}$ to $5 \times 10^{8}$ microbubbles $/ \mathrm{ml}$, with $90 \%$ of microbubbles $<8 \mu \mathrm{m}$ in diameter. Supplied as a lyophilized product in a septum-sealed vial, the contrast agent was reconstituted by injecting $5 \mathrm{ml}$ of saline through the septum, followed by manual agitation. Following a bolus injection, the contrast agent was circulated into the cardiovascular system, which lasted for up to $5 \mathrm{~min}$.

Equipment and examination. Conventional US and CEUS were performed using a CX50 system (Philips Medical 


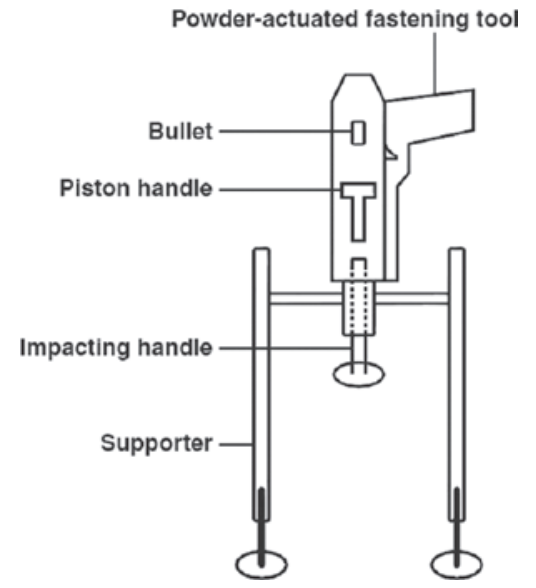

Figure 4. Construction plan of the impactor, consisting of a supporter, an impacting handle, a piston handle, a power bullet and a power actuated fastening tool. The impacting force was calculated based on the weight of the dog at $0.28 \mathrm{kN} / \mathrm{kg}$. In this experiment, the force was $4.8-5.6 \mathrm{kN}$ (the mean $5.3 \pm 0.3 \mathrm{kN})$.

Systems, Andover, MA, USA) at 0, 24, 48 and $72 \mathrm{~h}$. A L12-3 transducer with 3.0-12 MHz was also used in the current study.

CEUS employed the pulse inversion harmonic and the energy-modulated technique at a low acoustic power (a mechanical index of 0.07), which detects not only the nonlinear second harmonic response of microbubbles, but also the strong non-linear fundamental component. This process increased the signal-to-noise ratio by $15-20 \mathrm{~dB}$ and provided a much stronger contrast signal. In the current study, the pulse inversion harmonic and the energy-modulated technique were used to observe the sonographic appearance of the hematoma. The scan settings during the experiment (including the gain, the scanning depth and the time gain control) were optimized for each region independently. The focus was set on the deeper layer of the lesion examined. Meanwhile, conventional US were performed first, and SonoVue ${ }^{\mathrm{TM}}(0.025 \mathrm{ml} / \mathrm{kg})$ was administrated with a quick bolus through the accessory cephalic vein. Scanning began immediately after each injection, lasting for 3-5 min. Digital images were recorded as single-frame images and multiple cine loops on the hard disk of the scanner, for off-line analysis.

\section{Results}

Establishment of the model. Splenic hematoma was successfully induced on 9 dogs $(60.0 \%)$, failed on 4 (26.7\%), while 2 dogs (13.3\%) died. Consequently, the death was anatomically confirmed to have been caused by the bleeding after splenic rupture. CEUS showed low perfusion in the spleen, which was significantly reduced after $72 \mathrm{~h}$ for the dogs without splenic hematoma. Thus, the 6 dogs mentioned above were removed from the experiment. The remaining 9 dogs with splenic hematoma were used to analyze the data.

Observation of hematomas through conventional US, CEUS and CT. Conventional US showed a heterogeneous hypoechoic lesion in the spleen with a poorly defined margin. No significant change was observed after $24 \mathrm{~h}$ of observation. All the hema-

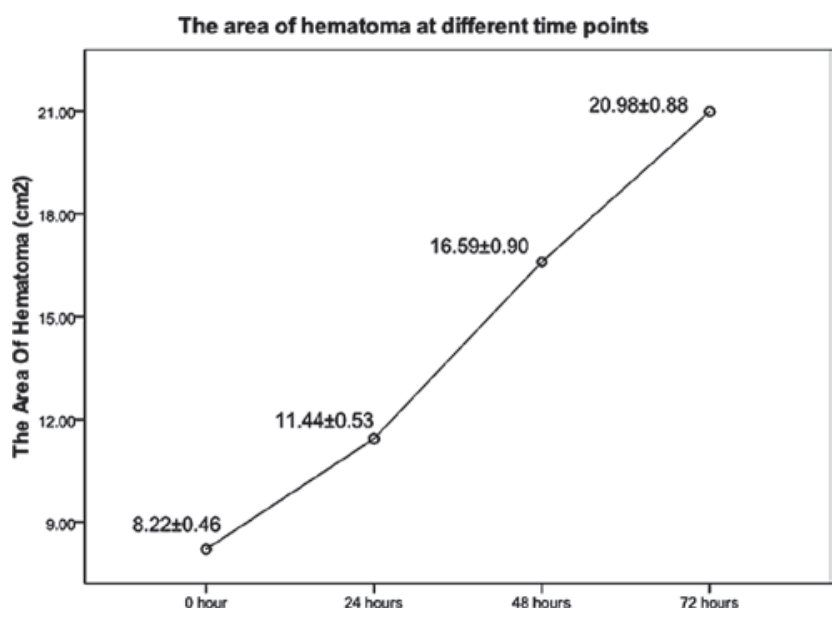

Figure 5. The area of the hematoma at different time-points. The area of the hematoma at 4 different time-points gradually increased.

toma lesions were clearly identified via CEUS after injecting SonoVue, which showed the lesions as anechoic perfusion defects in the arterial, portal and late phases, lasting for $\sim 5 \mathrm{~min}$. The areas of the hematoma at 4 different time-points gradually increased (Fig. 5). The CT examination performed before the rupture demonstrated that the volume of the spleen had increased with a round or oval shape of a lower density (Fig. 6).

Observation on hematomas after rupture by conventional US, CEUS and CT. Conventional US showed a slightly hyperechoic region, with an unclear boundary, intraperitoneal and perisplenic fluid. CEUS showed perisplenic hemoperitoneum, discontinued spleen capsule and large lamellar and irregular anechoic areas in the parenchyma with a clear margin. On the other hand, the CT examination showed the irregular and unenhanced areas in the spleen parenchyma with discontinued spleen capsule (Fig. 7).

Gross anatomy. Nine dogs underwent splenectomy, in which the gross specimen revealed a splenic parenchyma hematoma and varying degrees of rupture (Fig. 8).

None of the dogs had an adverse reaction during the CEUS examination, nor had any complications after the splenectomy, and all of them recovered rapidly.

\section{Discussion}

The etiology of delayed rupture is secondary to blunt trauma, such as falls, altercations, sports injuries and motor vehicle accidents (18). Countless theories have attempted to explain the mechanism of DSR, including the secondary hemorrhage and the delayed diagnosis of splenic injury (19-21). The widely accepted mechanism involves the formation of a subcapsular hematoma, which develops an increasing tension and eventually ruptures through the capsule, causing intraperitoneal bleeding (22).

Aside from ultrasonography, focused assessment with sonography for trauma (FAST) has emerged as a rapid and efficient method for the detection of hemoperitoneum in blunt abdominal injury patients (23). FAST has a comparable sensi- 

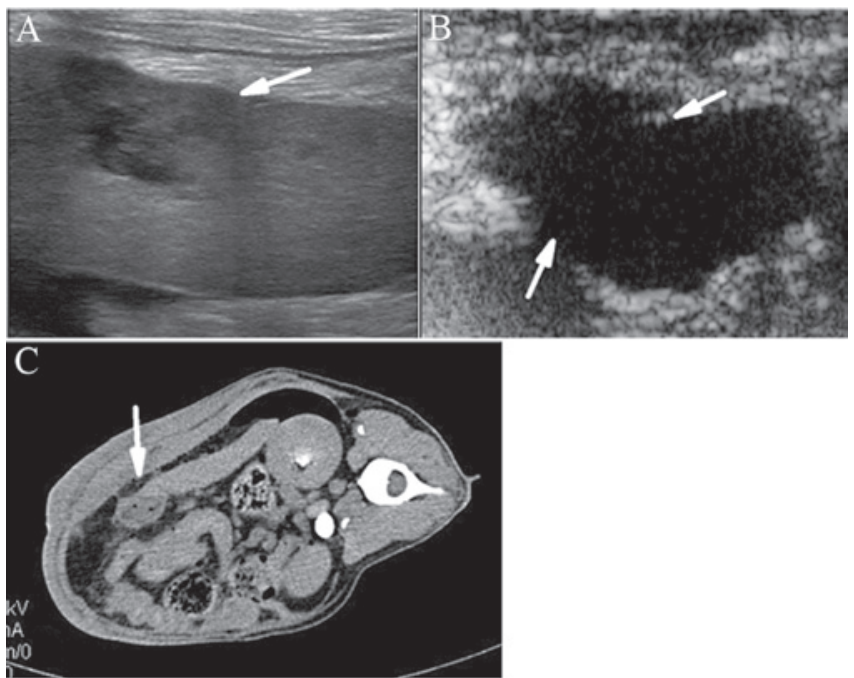

Figure 6. Observation of hematomas by conventional US, CEUS and CT. (A) Conventional US showed a heterogeneity hypoechoic region in the spleen with a poorly defined margin (white arrow). (B) The hematoma lesions were clearly identified by CEUS, which showed the lesions as anechoic perfusion defects in the arterial, portal, and late phases, lasting approximately $5 \mathrm{~min}$ (white arrow). (C) CT examination demonstrated that the volume of the spleen increased with a round or oval shape of a lower density (white arrow).

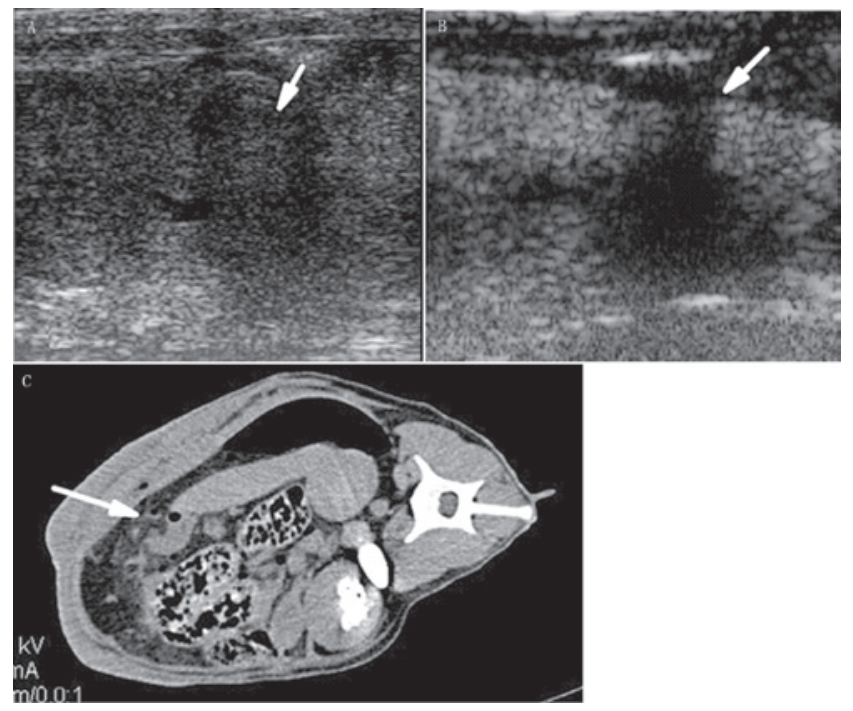

Figure 7. Observation of hematomas after rupture by conventional US, CEUS and CT. (A) Conventional US showed a slightly hyperechoic region, with an unclear boundary (white arrow). (B) CEUS showed perisplenic hemoperitoneum, discontinued spleen capsule and the large lamellar and irregular anechoic areas in the parenchyma with a clear margin (white arrow). (C) CT examination showed the irregular and unenhanced areas in the spleen parenchyma with discontinued spleen capsule (white arrow).

tivity and specificity for the detection of abdominal free fluid in patients with abdominal trauma due to its high sensitivity and availability $(7,8,24)$. However, in a previous study, conventional US detected only splenomegaly and irregular splenic border. Conventional US showed an irregular splenic border, discontinuity of the splenic capsule, perisplenic and intraperitoneal fluid after the hematoma was ruptured. However, in a 24-h observation interval, conventional US could not reliably determine the site of the hemorrhage, nor the active hemorrhage (9).

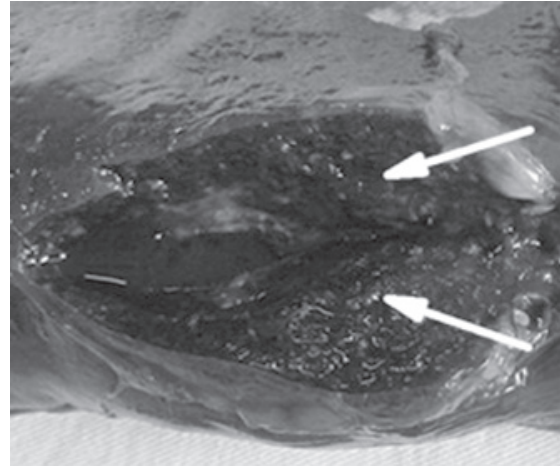

Figure 8. Gross specimen revealing splenic parenchyma hematoma and different degrees of rupture (white arrows).

Since the mid-1980s, CT scan has become a mainstay in the evaluation of intra-abdominal injury in hemodynamically stable patients, and has been proven to be efficient for trauma evaluation (10). The routine use of the CT scan can provide a more accurate assessment on the range and severity of the injury and the volume of bleeding, contributing to a more accurate diagnosis of splenic injury. Fagelman et al (25) described a patient, whose post-injury CT scans were normal but the repeated scan showed a splenic rupture caused by the secondary injury after $48 \mathrm{~h}$. In the current study, 2 of the 9 dogs $(22.2 \%)$ were normal in the first detection, but showed subcapsular hematomas and perisplenic fluid collection after the second scanning. The reason of the false negatives may be the included artifact or interference from surrounding tissues, making the injury difficult to detect, or an early CT scan taken before the subcapsular hematoma had bled enough (26).

CEUS demonstrates notable advantages in the evaluation of intra-abdominal injuries, and is superior to conventional US in diagnosing abdominal trauma (27-29). It also demonstrates the injury site as a non- and/or hypo-enhanced region with a clear extent and contrast material indicating an active hemorrhage. In the current study, CEUS showed the splenic hematomas and the significant development of the diameter of the hematomas within $48 \mathrm{~h}$. CEUS clearly showed the boundary and the range of the hematomas in all the dogs $(100 \%)$.

Compared with the CT scan, one of the advantages of CEUS is its rapid diagnosis of the hematoma lesions. In the past, patients usually needed to be transferred to the CT scanning room for examination and diagnosis. However, CEUS only needs 8-10 min to observe the injury, determine the grade and make appropriate triage decisions, whereas no investigations have accurately described the grade of the trauma when using conventional US. Another advantage of CEUS is its being free of radiation. In the current study, the dogs required over 4-fold the exposure time to the imaging detecter. However, 4-fold the exposure time to CEUS showed no adverse reactions, whereas 4-fold the exposure time to the radiation of the CT scan cannot be tolerated.

The animal model used in the current study has the following advantages: i) the modeling process was simple; ii) the splenic hematomas of the dogs had a long duration, with a stable status and strong comparability before and after the experiment was observed; iii) it can be used to verify the 
formation process of the DSR and the treatment of an experiment research in future trials.

The current experimental study has several limitations: i) it needs to be verified with a larger sample size, in order to obtain a more uniform number of dogs and splenic hematomas; ii) dogs have a strong hemostatic ability, which differs from splenic ruptures that commonly occur in humans.

In conclusion, the animal model of DSR is easy to fabricate, reliable for operation and can be evaluated using CEUS, which is more sensitive than conventional US and the CT scan. The DSR animal model has some similarities with actual clinical patients and may play an important role in clinical research, in view of being safe and effective.

\section{Acknowledgements}

The present study was supported by the National Natural Science Foundation of China (No. 81071279).

\section{References}

1. Foster RP: Delayed haemorrhage from the ruptured spleen. Br J Surg 57: 189-192 1970.

2. Schwartz SI, Shires GT and Spencer FC: Principles of Surgery. 5 th edition. McGraw-Hill Book Company, New York, NY, pp271-273, 1989.

3. Kluger Y, Paul DB, Raves JJ, Fonda M, Young JC, Townsend RN and Diamond DL: Delayed rupture of the spleen - myths, facts, and their importance: case reports and literature review. J Trauma 36: 568-571, 1994

4. Benjamin CI, Engrav LH and Perry JF Jr: Delayed rupture or delayed diagnosis of rupture of the spleen. Surg Gynecol Obstet 142: 171-172, 1976.

5. Berlatzky Y, Shiloni E, Anner H and Weiss Y: 'Delayed rupture of the spleen' or delayed diagnosis of the splenic injury? Isr J Med Sci 16: 659-664, 1980.

6. Cogbill TH, Moore EE, Jurkovich GJ, Morris JA, Mucha P Jr, Shackford SR, Stolee RT, Moore FA, Pilcher S, LoCicero R, et al: Nonoperative management of blunt splenic trauma: a multicenter experience. J Trauma 29: 1312-1317, 1989.

7. Richards JR, Schleper NH, Woo BD, Bohnen PA and McGahan JP: Sonographic assessment of blunt abdominal trauma: a 4-year prospective study. J Clin Ultrasound 30: 59-67, 2002.

8. Shanmuganathan K, Mirvis SE, Sherbourne CD, Chiu WC and Rodriguez A: Hemoperitoneum as the sole indicator of abdominal visceral injuries: a potential limitation of screening abdominal US for trauma. Radiology 212: 423-430, 1999.

9. Yoshii H, Sato M, Yamamoto S, Motegi M, Okusawa S, Kitano M, Nagashima A, Doi M, Takuma K, Kato K and Aikawa N: Usefulness and limitations of ultrasonography in the initial evaluation of blunt abdominal trauma. J Trauma 45: 45-50, 1998.

10. Becker CD, Mentha G and Terrier F: Blunt abdominal trauma in adults: role of $\mathrm{CT}$ in the diagnosis and management of viscera injuries. Part 1: liver and spleen. Eur Radiol 8: 553-562, 1998.
11. Richards JR and Derlet RW: Computed tomography for blunt abdominal trauma in the ED: a prospective study. Am J Emerg Med 16: 338-342, 1998.

12. Xie H, Wolf RF, Burke AP, Gustafson SB, Gregory KW and Prahl SA: Concentrated albumin as a biological glue for hemorrhage control on hepatic resection with argon beam coagulation. J Biomed Mater Res B Appl Biomater 71: 84-89, 2004.

13. Li Z, Dong XZ, You FS, Zhao DQ, Lin X, Zhang GP and Zheng WS: Establishment of animal model of intraperitoneal bleeding in rabbits. Chin J Comparative Med 4: 217-219, 2006.

14. Leen E, Angerson WJ, Yarmenitis S, Bongartz G, Blomley M, Del Maschio A, Summaria V, Maresca G, Pezzoli C and Llull JB: Multi-centre clinical study evaluating the efficacy of SonoVue (BR1), a new ultrasound contrast agent in Doppler investigation of focal hepatic lesions. Eur J Radiol 41: 200-206, 2002.

15. von Herbay A, Vogt C, Willers R and Häussinger D: Real-time imaging with the sonographic contrast agent SonoVue: differentiation between benign and malignant hepatic lesions. J Ultrasound Med 23: 1557-1568, 2004.

16. Strobel D, Kleinecke C, Hänsler J, Frieser M, Händl T, Hahn EG and Bernatik T: Contrast-enhanced sonography for the characterisation of hepatocellular carcinomas-correlation with histological differentiation. Ultraschall Med 26: 270-276, 2005.

17. Albrecht T, Hohmann J, Oldenburg A, Skrok J and Wolf KJ: Detection and characterisation of liver metastases. Eur Radiol 14: 25-33, 2004.

18. Dang C, Schlater T, Bui H and Oshita T: Delayed rupture of the spleen. Ann Emerg Med 19: 399-403, 1990.

19. Zabinski EJ and Harkins HN: Delayed splenic rupture: A clinical syndrome following trauma. Arch Surg 46: 186-212, 1943.

20. Sizer JS, Wayne E and Frederick PL: Delayed rupture of the spleen. Arch Surg 92: 362-366, 1966.

21. Olsen WR and Polley TZ Jr: A second look at delayed splenic rupture. Arch Surg 112: 422-425, 1977.

22. Farhat GA, Abdu RA and Vanek VW: Delayed splenic rupture: real or imaginary? Am Surg 58: 340-345, 1992.

23. Scalea TM, Rodriguez A, Chiu WC, Brenneman FD, Fallon WF Jr, Kato K, McKenney MG, Nerlich ML, Ochsner MG and Yoshii H: Focused assessment with sonography for trauma (FAST): results from an international consensus conference. J Trauma 46: 466-472, 1999.

24. Benya EC, Lim-Dunham JE, Landrum O and Statter M: Abdominal sonography in examination of children with blunt abdominal trauma. AJR Am J Roentgenol 174: 1613-1616, 2000.

25. Fagelman D, Hertz MA and Ross AS: Delayed development of splenic subcapsular hematoma: CT evaluation. J Comput Assist Tomogr 9: 815-816, 1985.

26. Gamblin TC, Wall CE Jr, Royer GM, Dalton ML and Ashley DW: Delayed splenic rupture: case reports and review of the literature. J Trauma 59: 1231-1234, 2005.

27. Li YK, Zhou XD, Zhang J, et al: Contrast-enhanced ultrasonography for evaluation of blunt hepatic trauma with active bleeding. Chin J Med Ultrason 14: 618-620, 2005.

28. Spinazzi A: Emerging clinical applications for contrast-enhanced ultrasonography. Eur Radiol 11: E7-E12, 2001.

29. Catalano O, Lobianco R, Raso MM and Siani A: Blunt hepatic trauma: Evaluation with contrast-enhanced sonography: Sonographic findings and clinical application. J Ultrasound Med 24: 299-310, 2005 . 